ÉGYPTE

monde arabe

\section{Égypte/Monde arabe}

30-31 | 1997

Les visions de l'Occident dans le monde arabe

\title{
De la mort de l'éthique au deuil de l'optimum
}

L'Occident économique au miroir de l'« Économie islamique »

\section{François Ireton}

\section{(2) OpenEdition}

\section{Journals}

Édition électronique

URL : https://journals.openedition.org/ema/1651

DOI : 10.4000/ema.1651

ISSN : 2090-7273

Éditeur

CEDEJ - Centre d'études et de documentation économiques juridiques et sociales

Édition imprimée

Date de publication : 30 septembre 1997

Pagination : 251-274

ISSN : 1110-5097

Référence électronique

François Ireton, «De la mort de l'éthique au deuil de l'optimum », Égypte/Monde arabe [En ligne], 30-31 | 1997, mis en ligne le 08 juillet 2008, consulté le 07 juillet 2022. URL : http://journals.openedition.org/ ema/1651 ; DOI : https://doi.org/10.4000/ema.1651

Ce document a été généré automatiquement le 7 juillet 2022

Tous droits réservés 


\title{
De la mort de l'éthique au deuil de l'optimum
}

\author{
L'Occident économique au miroir de l'« Économie islamique »
}

François Ireton

1 On s'interrogera ici sur certains contenus explicites et certaines structures implicites des représentations que construisent, à propos des réalités économiques «occidentales», des intellectuels musulmans contemporains, pour la plupart universitaires, produisant des discours concernant ce qu'ils nomment eux-mêmes l'« économie islamique ». Par « réalités économiques occidentales », l'on entendra ce que ces intellectuels considèrent comme caractérisant, dans les sphères de la production et de l'échange des biens et des services ainsi que de la répartition des revenus et richesses, l'Occident géographique proprement dit, mais aussi d'autres régions du globe, dans la mesure où certaines des structures économiques de ces dernières tirent leur origine de l'intervention directe ou indirecte de pays appartenant à l'Occident géographique, et se reproduisent ou se transforment, dans le cadre de l'économie mondiale, en rapport avec eux (il s'agit de rapports d'interdépendance asymétrique, selon la plupart de ces auteurs). Par «discours concernant l'économie islamique ", l'on comprendra - d'une manière intentionnellement restrictive dont on s'expliquera plus loin - le corpus des écrits qui se donnent pour fonction d'exposer ce que devraient être les pratiques, organisations concrètes et structures objectives socioéconomiques qui caractériseraient une société contemporaine cherchant à appliquer les principes éthico-religieux généraux comme spécifiquement économiques de l'islam et à mettre en place, afin d'«islamiser» son système économique, et au prix d'adaptations aux « réalités contemporaines ", les institutions spécialisées mentionnées dans les textes canoniques. Ce sont quelques représentations de l'économie et des sociétés « occidentales » (au sens retenu plus haut), figurant dans ce type de discours sur l'économie islamique, à titre de descriptions d'états de chose à transformer ou de contre-exemples à ne pas imiter, qui constitueront l'objet d'étude de ce travail. L'on cernera d'abord les caractéristiques particulières du type de discours retenu, parmi l'énorme masse de ceux qui concernent l'«économie islamique " et émanent de différents groupes d'acteurs plus ou moins directement concernés par l'« islamisation 
de l'économie" (religieux, universitaires de diverses disciplines, juristes, hommes politiques, hauts fonctionnaires, banquiers, hommes d'affaires et autres praticiens de l'économie). L'on dégagera ensuite, à partir d'un ouvrage considéré comme un bon exemple du type de discours visé, les modes de construction des représentations, à la fois analytiques et normatives - donc critiques - qui y sont données de l'Occident économique, construction mettant en œuvre un double système de références éthiques et théoriques. Enfin, l'on exposera les grandes articulations de la vision que l'auteur de l'ouvrage examiné livre de ce qui constitue pour lui la matrice historique des réalités économiques occidentales contemporaines : le capitalisme libéral, dont la mise en place progressive s'est effectuée en interaction avec le processus de sécularisation de l'Occident.

\section{Discours d'économie islamique}

\section{Les trois registres de l'Économie universitaire}

2 Comme on le sait, dans certaines langues comme le français ou l'arabe, le mot " économie » (iqtisâd) peut renvoyer soit aux « réalités » économiques (on emploiera ici ce mot sans majuscule initiale), soit à divers discours les concernant, considérés comme relevant d'un savoir spécialisé (on écrira "Économie», l'expression "Science(s) Économique(s) », 'ilm al-iqtisâd, étant aussi employée dans ce sens). L'économie consiste, selon la définition savante dite "substantielle », en l'ensemble des pratiques, rapports sociaux, institutions et représentations «spontanées" liés à la production, la circulation, la répartition et la consommation des richesses. Il semble utile de rappeler d'emblée, puisqu'il va s'agir d'« économie islamique » et de capitalisme libéral, que les réalités économiques sont, selon les sociétés et les phases de leur évolution historique, plus ou moins différenciées et plus ou moins autonomes - embedded ou disembedded, selon la terminologie de K. Polanyi - par rapports aux autres registres de pratiques (politique, religieux, etc.) et donc plus ou moins et diversement thématisées et catégorisées comme telles dans les systèmes de représentations produits par ces sociétés.

3 Les discours concernant les réalités économiques, quant à eux - discours dont la genèse et la structure sont en partie liées au degré de différenciation externe et interne du champ économique - consistent en énoncés habituellement classés par l'épistémologie de l'Économie en "positifs », normatifs (ou doctrinaux : on parle des " grandes doctrines économiques ») et techniques (cette tripartition est empruntée à John Neville Keynes, qui distingue : «la science positive... corps de savoir systématisé concernant ce qui est ; la science normative ou régulatrice... corps de savoir systématisé discutant des critères de ce qui devrait être ; et l'art... système de règles pour l'obtention d'une fin donnée.» ${ }^{1}$. Les énoncés normatifs :

1. élaborent les principes dont l'application à la sphère économique est prescrite,

2. décrivent un état idéal du champ économique, c'est-à-dire ce qu'il serait s'il se conformait aux principes prescrits, divers quant à leur nature (éthico-religieuse, comme "l'égalité devant Dieu ", éthico-politique, comme l'équité, ou simplement économique, comme l'efficience) et spécifiés, si nécessaire, en vue de leur application au champ économique, c) évaluent l'écart entre cet état idéal du champ économique et son état réel analysé par la "science positive », ce qui permet le diagnostic des «facteurs négatifs » responsables de cet état de fait. Les énoncés programmatiques et techniques exposent les méthodes permettant 
la mise en œuvre des mesures prises en vue de la réduction de cet écart et déductibles des modèles d'interrelation entre les faits que construit la "science positive », laquelle permet aussi de tester l'efficacité et l'applicabilité de ces mesures. Les énoncés " positifs », quant à eux, sont soit purement théoriques (cf. les théories hypothético-déductives proposées par l'«économie pure », telle celle de l'équilibre général), soit théoriques et analytiques : ils mettent en œuvre une théorie, toujours modifiable, en vue de rendre compte d'états ou de changements d'état du champ économique, appréhendés et construits comme objets de savoir à l'aide des concepts et modèles de la théorie.

\section{Les cinq contenus de l'Économie islamique}

Pour appliquer aux discours d'Économie islamique ces distinctions épistémologiques, il est nécessaire de s'interroger préalablement sur la variété des contenus de ces discours. Schématiquement, sous le nom d' "Économie islamique », l'on a affaire à cinq types de contenus (dont tous ou certains d'entre eux peuvent figurer ou non ensemble dans les discours considérés comme en relevant) :

a) les premiers consistent en exégèses des textes canoniques - le Coran, la Sunna (hadith) et des textes de figh - visant à restituer la définition, d'une part, des grands principes éthiques de l'islam devant être appliqués à l'ordre économique de toute société se voulant islamique et, d'autre part, des institutions économiques - ainsi que de leur fonctionnement - à mettre en place dans de telles sociétés afin de mettre en pratique ces principes ;

6 b) d'autres consistent en descriptions de la société des premiers temps de l'islam (contemporaine du Prophète et des quatre premiers califes), appréhendée à travers des textes anciens, canoniques ou non, descriptions visant à restituer un état des pratiques économiques de l'époque pouvant éventuellement servir de modèle à des pratiques contemporaines - moyennant un travail d'adaptation -, pourvu que ces pratiques d'origine soient reconnues, après discussion, conformes aux principes de l'islam en la matière ;

7 c) un très vaste corpus d'énoncés, cités en introduction, se donne pour fonction d'exposer ce que seraient (ou doivent être) les pratiques, organisations concrètes et structures objectives socio-économiques qui caractériseraient, selon leurs auteurs, une société contemporaine cherchant, d'une part, à « appliquer » en matière économique les principes éthico-religieux généraux figurant dans les textes considérés comme canoniques par les autorités de l'islam (comme la « justice distributive ») et à respecter les injonctions spécifiquement économiques contenues dans ces textes (comme l'interdiction du riba) et, d'autre part, à mettre en place, afin d'«islamiser » son système économique, les institutions spécialisées mentionnées dans ces textes moyennant, le plus souvent, leur adaptation aux « réalités contemporaines » jugées non contraires aux principes de l'islam - ou des institutions qui n'y figurent pas mais dont la création, formant matière à discussion, est jugée par ces auteurs nécessaire à la poursuite du même but ;

8 d) certains énoncés décrivent des réalités économiques considérées comme n'entretenant pas de relations particulières avec l'islam et cherchent à les évaluer du point de vue de ce dernier ;

9 e) enfin, l'on rencontre une dernière catégorie de contenus qui consistent en descriptions de réalités économiques explicitement reliées à l'islam (des institutions, le 
plus souvent, telles que les Banques dites islamiques) qui peuvent faire aussi i'objet d'évaluation en terme de conformité à l'idéal islamique en la matière² 2 .

\section{Registres et contenus : croisements}

10 Les énoncés véhiculant ces contenus articulent de manière différente l'analyse positive, les assertions normatives et les propositions techniques. Les contenus du premier type correspondent à des énoncés explicitement normatifs, concernant la recherche des principes. L'exposé des contenus des second, quatrième et cinquième types mobilise des énoncés positifs (la description des réalités économiques passées et contemporaines, reliées ou non, dans ce dernier cas, à l'islam) et normatifs, concernant l'évaluation de ces réalités du point de vue de l'islam. Quant aux contenus du troisième type, ils supposent tout à la fois des énoncés normatifs (description d'un état idéal du monde économique), des énoncés programmatiques et techniques (la mise en oeuvre des mesures à prendre pour atteindre cet idéal) et d'autres, enfin, positifs mais d'une nature particulière, décrivant des mondes économiques possibles, après application de ces mesures (mondes qui constituent un test de l'efficacité de ces dernières).

11 L'ensemble des énoncés de l'Économie islamique recouvre donc l'ensemble des registres rencontrés dans les Sciences Économiques « séculières ». Il est en effet clair, pour de nombreux économistes réfléchissant sur l'épistémologie de leur discipline ${ }^{3}$ que, non seulement de nombreuses «branches" de cette dernière sont normatives par nature (la New Welfare Eonomics ou la théorie des choix sociaux, par exemple), mais encore qu'une partie importante de l'armature conceptuelle de la théorie néoclassique l'est également; en particulier, la notion d'« efficience», qui est au cœur de cette théorie, est implicitement normative (elle décrit bien un état "idéal», d'après les tenants de cette dernière), mais seule une approche critique de ses fondements peut en révéler le caractère normatif, alors que la notion d'équité se donne d'emblée comme éthique et renvoie à de nombreuses définitions argumentées dans un cadre philosophique. Inversement, si l'Économie islamique apparaît immédiatement comme normative, ses discours comportent de nombreux énoncés analytiques (dès que des contenus de type « $\mathrm{c}$ », « $\mathrm{d}$ » ou « $\mathrm{e}$ » sont abordés); ces derniers supposent l'appel à divers courants théoriques contemporains relevant de l'Économie "séculière ", parmi lesquels certains sont plus sollicités que d'autres (la macro-économie post-keynésienne par exemple, ou divers courants institutionnalistes, davantage - comme on peut l'imaginer - que les élaborations les plus récentes dérivées du paradigme néoclassique $)^{4}$.

\section{Une Économie islamique universitaire}

12 L'objet dont on s'est fixé l'exploration, les visions de l'Occident économique, relève clairement des énoncés véhiculant des contenus de type « $d$ » : analyse et évaluation, d'un point de vue islamique, de réalités socio-économiques considérées comme n'entretenant aucune relation particulière avec l'islam. Le corpus des énoncés concernant des regards portés, d'un tel point de vue, sur l'économie occidentale telle qu'on l'a définie plus haut, est cependant considérable: ces énoncés sont le fait d'hommes politiques, de journalistes, d'hommes de religion et essayistes, mais il se trouve que les productions de ces derniers, moins techniques et donc plus accessibles que celles que l'on va examiner, sont mieux relayées par les médias nationaux (presse, 
télévision), s'insèrent largement dans des débats publics à l'intérieur des pays musulmans et de ce fait sont mieux connues à l'extérieur de ces derniers, où elles ont fait l'objet de nombreuses analyses, en particulier lorsqu'elles contiennent des prises de position concernant la mise en place de réformes visant une «islamisation de l'économie ", souvent redoutée des puissances occidentales ou lorsqu'elles constituent des accusations ou des plaidoyers liés à quelques scandales éclatant dans des institutions financières se donnant comme « islamiques ».

L'on a choisi - sans qu'il entre dans ce choix aucun jugement de valeur-d'examiner ici un exemple de vision très construite et insérée dans un contexte discursif particulier, celui d'ouvrages universitaires qui, se contentant de reprendre à leur compte les grands principes éthiques de l'islam en matière économique et ne cherchant à évaluer ni le caractère islamique exemplaire de l'ordre économique des premiers temps de l'islam, ni celui des institutions "islamiques» (essentiellement des banques) actuellement existantes, livrent des analyses et des évaluations des divers grands systèmes économiques étrangers à l'islam qu'ils mettent en regard avec des élaborations théoriques relevant de contenus de types «c» («modélisation» de systèmes économiques à mettre en oeuvre dans les sociétés contemporaines et intégrant les principes éthiques et les propositions institutionnelles de l'islam en matière d'économie). L'on a plus spécifiquement examiné un petit ensemble d'ouvrages théoriques - dont les analyses empiriques ne sont bien sûr pas absentes - rédigés en langue anglaise et dont les auteurs sont économistes de formation. Ayant quasiment tous fait leur études dans leur pays d'origine (Moyen-Orient arabe, Pakistan, actuel Bangladesh, Malaisie) et en Occident - États-Unis en particulier - où ils ont en général obtenu leur doctorat sur un sujet relevant d'une branche bien sûr "séculière " (mais non nécessairement des plus " classiques») des sciences économiques ${ }^{5}$, ils enseignent aussi bien dans des universités américaines que dans celles de pays musulmans et sont souvent experts ou conseillers auprès d'institutions économiques publiques, nationales ou internationales, nommément islamiques ou du moins relevant de pays islamiques.

Dans le cadre de ce travail exploratoire, aucune tentative de construction d'un « champ de la production universitaire en Économie islamique » n'a été entreprise, celle-ci supposant préalablement le rassemblement de nombreuses caractéristiques sociologiques de leurs auteurs et des institutions auxquelles ils appartiennent. Ceux dont on a consulté les écrits et les deux auteurs dont on a lu systématiquement certains des ouvrages ${ }^{6}$ sont quant à eux, quasi inconnus dans la sphère des area studies portant sur le «monde musulman » (leur nom n'apparait pas dans les articles traitant des idées ou des faits d'économie islamique parus dans les grandes revues scientifiques spécialisée dans cette "aire culturelle»). Par contre, leur travaux sont connus et commentés par certains économistes « séculiers »-y compris de renommée mondiale comme Kenneth Boulding - pour qui le modèle néoclassique dans ses différents avatars ne constitue pas, comme le disait Sartre du marxisme, l'« horizon indépassable de notre temps $»^{7}$.

\section{Économie des visions de l'Occident économique}

15 Dans le texte sur lequel porte le travail présenté ici, la vision de l'Occident économique présentée par l'auteur est largement développée et joue un rôle stratégique dans l'argumentation et l'exposition des contenus de l'«Économie islamique», alors que 
dans de nombreux autres textes, ne sont livrées que des allusions éparses et ponctuelles à certains aspects de ce qui est considéré comme appartenant à la réalité économique "occidentale ", allusions insuffisantes pour reconstituer une "vision " à proprement parler. L'inconvénient du choix effectué ici réside dans le fait qu'en choisissant d'en analyser une qui est largement développée et comprend des analyses argumentées - se voulant démonstratives et établissant des liaisons causales ou fonctionnelles entre différents aspects des réalités, économiques et non économiques, considérées comme caractérisant l'Occident -, on laisse de côté les visions fragmentaires sans doute majoritaires et plus clairement "stéréotypées ». Cependant, quel que soit le niveau d'élaboration de ces représentations, elles ont au moins ceci en commun de toujours constituer des visions normatives, évaluatrices et critiques, élaborées d'abord d'un point de vue éthique, et ceci qu'il s'agisse d'économie ou d'autres registres de la réalité sociale. Enfin, une «stratégie argumentative » très pratiquée par les auteurs de discours d'Économie islamique relevant du type pris en compte ici et comportant des visions de l'Occident économique, consiste à emprunter une grande partie de leurs analyses critiques à des auteurs occidentaux eux-mêmes, contemporains pour la plupart, abondamment cités et pouvant se situer d'un pôle à l'autre du spectre des options idéologiques «occidentales». S'expriment ainsi fréquemment, dans ces visions critiques, des préoccupations et des jugements éthicoéconomiques émanant de courants de pensée "transnationaux ", ainsi que des prises de positions qui dépassent de loin un horizon que certains supposent limité aux problèmes de la «ré-islamisation» des sociétés dites musulmanes. Loin d'être un quelconque "retour à », ce corpus de discours d'Économie islamique est donc strictement contemporain.

\section{La construction d'une vision critique (Islam and the economic challenge, M. U. Chapra)}

\section{Occident économique et principes éthico-économiques de l'islam}

L'on se bornera ici à analyser la vision que l'auteur donne d'une partie de l'Occident économique, composée de l'Europe de l'Ouest et de l'Amérique du Nord. Cet ensemble est caractérisé selon lui, d'une part par l'« épanouissement » du libéralisme, tant sur le plan doctrinal (chez les théoriciens des $\mathrm{XVIII}^{\mathrm{e}}$ et $\mathrm{XIX}^{\mathrm{e}}$ siècles) que sur celui des " réalités » économiques (le capitalisme libéral du XIX ${ }^{\mathrm{e}}$ siècle et du début du XX $\mathrm{XX}^{\mathrm{e}}$ siècle) et, d'autre part, par son évolution, dès les années trente de ce siècle, vers un capitalisme "encadré » par le Welfare State. Ces deux périodes font l'objet des premier et troisième chapitres (intitulés respectivement The Limits of Capitalism et The Crisis of the Welfare State) séparés par un autre consacré à l'« échec du socialisme » (The Retreat of Socialism) et suivis d'un quatrième consacré à révolution économique des "pays en développement» (intitulé The inconsistency of Development Economies). Alors que le premier chapitre se clôt sur la fin du «laissez-faire » (en français dans le texte), doctrine et réalités économiques dont les développements y font l'objet d'une longue analyse critique, les trois chapitres suivants se terminent sur le constat (face aux contradictions, aux insuffisances et finalement aux échecs du Welfare State, du «socialisme » et des modèles de développement adoptés par les pays du tiers monde) de l'appel au retour vers un capitalisme libéral (néolibéralisme), dont les fondements 
éthiques et les réalisations socio-économiques ont été invalidés d'avance par les analyses du premier chapitre. Le partiel et sélectif néolibéralisme de crise de longue durée caractérisant actuellement l'Europe de l'Ouest, l'ultralibéralisme aux mains d'une ex-nomenklatura avidement reconvertie dans l'affairisme sévissant en Europe de l'Est, le libéralisme doctrinaire prônant la déréglementation et l'ajustement structurel imposés par le "consensus de Washington" aux pays les moins développés et sa version sud-est asiatique conquérante et liquidatrice de processus de développement moins inégalitaires qu'ailleurs, toutes ces formes nouvelles de retour à ce qui se voudrait une pure économie (et société) de marché, bien que différant par plusieurs aspects du "libéralisme classique ", ne semblent guère à l'auteur garantir davantage que ce dernier une croissance économique socialement et éthiquement acceptable.

Cette dernière est nécessaire tant que l'accès aux biens indispensables à la satisfaction des basic needs, matériels et immatériels, définis comme partie intégrante de la hayâttayyiba (la vie bonne - et non pas la «bonne vie ») ou falâh (le bien-être), n'est pas assuré. Cette croissance doit se réaliser dans et par l'efficience (efficiency), qui ne peut constituer pour l'auteur une garantie de «bon fonctionnement » de l'économie, comme c'est le cas dans le corpus théorique néoclassique. Dans ce dernier en effet, l'efficience, découlant des comportements maximisateurs des "homo economicus", représente l'idéal de la rationalité économique, accompli dans l'état d'équilibre général. Or celui-ci correspond à un " équilibre de Pareto ", défini comme une configuration de répartition des revenus telle qu'il n'y est pas possible d'accroître ceux des plus démunis sans diminuer ceux des mieux dotés, opération qui ne peut s'effectuer que par le recours à des mesures volontaristes et étatiques de réallocation entraînant nécessairement la rupture de l'état d'équilibre. Qu'une telle configuration de la répartition des revenus, grosse de multiples formes d'inégalités socio-économiques, puisse constituer un "optimum» apparaît comme inadmissible à l'auteur pour qui l'amélioration des conditions de vie des plus pauvres nécessite, entre autres mesures, le sacrifice d'une part du bien-être des plus riches. L'efficience néo-classique, souvent associée à une répartition très inégalitaire, ne saurait ainsi en rien assurer une croissance éthiquement acceptable. L'efficience - que prône l'auteur parce qu'elle constitue une condition de la croissance garantissant un niveau de production adéquat de biens essentiels - est valorisée comme permettant d'éviter l'isrâf, le gaspillage des ressources naturelles (et des biens produits et consommés) que le principe de khilâfa (de «vicegérance ») de l'homme sur le monde créé par Dieu lui enjoint d'éviter, compte tenu de sa responsabilité vis-à-vis des plus démunis pour qui ces ressources et biens sont indispensables, mais aussi vis-à-vis des générations futures. Ce même principe, joint à celui de 'adâla (d'emblée traduit par l'auteur par equity), implique l'unité fondamentale de l'humanité, l'exigence de fraternité et de solidarité universelle, ainsi que l'égalité de droit de tous les êtres humains. La croissance doit donc s'effectuer non seulement dans l'efficience ainsi conçue mais dans l'équité (equity); celle-ci se réalise de deux manières: par la suppression de toute forme de dhulm (injustice, oppression, exploitation) économique; positivement, d'une part, par la possibilité que la société doit donner à tout être humain de pourvoir à sa subsistance et à celle de sa famille par l'obtention d'un revenu et, d'autre part, par un système de distribution et de redistribution des revenus et des richesses qui s'accorde avec le contenu donné à la notion d'équité. La croissance ainsi conçue n'est pas une fin en soi et n'est souhaitable que tant que les besoins vitaux et ceux qui permettent la «vie bonne» (selon la définition qui peut en être donnée, pour chaque époque, en accord avec les valeurs 
fondamentales de l'islam) ne sont pas satisfaits équitablement pour l'ensemble des humains. Sa poursuite n'est justifiée que si elle mène sans gaspillage à ce résultat.

L'on ne peut ici explorer en détail l'impressionnant travail de "traduction », effectué par des théoriciens de l'Économie islamique comme Chapra et Choudhury, de ce qu'ils considèrent comme étant les principes éthico-économiques de l'islam, traduction dont la mise en œuvre contemporaine suppose un travail d'interprétation (ijtihâd) et de formalisation dans un cadre théorique adéquat et qu'ils effectuent dans le langage de conceptions économiques contemporaines visant l'universalisme et l'accessibilité, mais resituées précisément et de manière critique parmi les philosophies éthicoéconomiques « séculières » existantes et les théories économiques dites positives ${ }^{8}$. C'est $^{\prime}$ en rapport avec cette interprétation des principes éthico-économiques de l'islam (dont la description succincte était donc nécessaire) exposée dans le premier chapitre de la deuxième partie de l'ouvrage (respectivement intitulés the islamic Worldview et The islamic Response), que l'auteur élabore sa vision critique de l'évolution des systèmes économiques occidentaux (capitalisme libéral, capitalisme encadré par le Welfare State, socialisme - dans ses diverses versions -éolibéralismes contemporains) et des modèles de développement mis en œuvre dans les pays du tiers monde, dont il tente de montrer qu'ils ne sont que l'adaptation, dans des contextes particuliers qui les spécifient et leur laissent moins de chance encore de réussite, des diverses formes de capitalisme et de socialisme examinés précédemment. Les conditions et les moyens (y compris institutionnels) d'application concrète de ces principes éthico-économiques « retraduits » sont examinés dans le reste de la seconde partie de l'ouvrage. On l'aura remarqué, le langage et le cadre théorique utilisés pour cette "traductionactualisation » des principes \{basic needs, growth with redistribution, efficiency and equity, poverty alleviation, etc.) est celui des théories du développement à la fois non néolibérales et non marxistes qui ont eu cours à la fin des années soixante, pendant les années soixante-dix et au début des années quatre-vingt, et dont la Banque mondiale, l'Unicef et l'Organisation internationale du travail furent d'importants vecteurs. Les objectifs réalisant l'application concrète de ces principes n'ont pas été atteints, constate l'auteur; ils ne pouvaient l'être, puisque certaines conditions préalables, d'ordres éthico-social, politique et religieux n'étaient pas remplies, conditions dont l'islam propose une représentation synthétique sur la base de laquelle il faut en partie imaginer les modalités de leur réalisation, seulement esquissées dans le corpus des textes canoniques'.

Que ces principes soient traduits en un langage économique qui peut apparaître comme « daté » n'invalide pas, pour l'auteur, l'importance de leur application. Par ailleurs, que les exigences - ou plutôt les souhaits - d'équité, de redistribution et d'allègement de la pauvreté soient cités, à titre subsidiaire, par les organismes fers de lance de l'ajustement structurel et de la déréglementation ne doit pas faire illusion: le néolibéralisme dans ses présupposés individualistes constitue la négation même des conditions de réalisation de ces objectifs, non pas secondaires mais prioritaires, réalisation qui n'est en rien automatiquement liée à une forte croissance et sans laquelle la résorption de la dette extérieure et la restauration des " grands équilibres » sont simplement dénuées de sens. Si l'auteur vise la mise en place progressive des conditions de l'application des principes éthico-économiques de l'islam de façon prioritaire dans les pays dits musulmans -appartenant quasiment tous aux «pays en développement» -précisément par le biais d'une « ré-islamisation» de ceux-ci, il ne 
considère pas qu'ils aient sur ce point un handicap favorable par rapport aux autres pays :

«The Muslim countries are not different. They are faced with the same problems as other countries. Theyare, however, thoughtlessly following the Westin everything and committing the same mistake of looking only at the symptoms. No serious effort is being made to analyse the underlying source of their problems and to determine a proper strategy for solving them in the light of their own value system. »(Chapra, 1985, p. 20)

\section{La théorie néoclassique comme pensée (très) sauvage}

"... Thoughtlessly following the West in eveything", là se situe pour l'auteur l'un des fondements de l'incapacité actuelle de l'ensemble des sociétés, musulmanes ou non, d'inventer des structures économiques susceptibles d'incarner les principes développés plus haut, sur lesquels chacun, musulman ou non, devrait s'accorder. L'« Ouest », sous ses aspects économiques, c'est le capitalisme, et ce l'est d'autant plus qu'apparaît un nouveau consensus, dans les sphères politiques et économiques dominantes de différentes parties du monde, quant à la nécessité d'un retour à son « existence pure ", sous sa forme intégralement libérale. La vision critique que l'auteur livre de l'Occident économique se focalise donc d'abord sur le capitalisme à l'« état pur ", dans sa version idéal-typique intégralement libérale, dont il affirme qu'elle n'a jamais historiquement existé sous cette stricte forme (Chapra, 1992, p.17). Le fait qu'il soit économiste explique sans doute qu'il semble d'abord appréhender le capitalisme libéral à travers les catégories du courant théorique dominant, dit néoclassique, qui a construit de ce système une vision savante devenue vulgate, dans le but affirmé d'en mettre à jour la logique. Mais d'emblée, un décalage apparaît au niveau même du vocabulaire : l'auteur écrit "capitalisme" (libéral) là où les tenants de la théorie néoclassique parlent d'« économie de marché ». Cette dissonance semble déjà, à elle seule, indiquer le fait que la théorie néoclassique ne sera nullement reprise comme grille d'analyse "scientifique » de ce qui constitue la structure centrale de l'Occident économique. Se situant dans une démarche normative, donc évaluatrice et critique sur le plan éthique (et non dans une démarche e " conquête de la vérité ", visant à dévoiler les "ressorts cachés " du système), l'auteur-il semble important de le noter - ne confirme ni ne conteste explicitement, du point de vue de sa pertinence analytique, la théorie néoclassique ${ }^{10}$. Visant à porter un jugement de valeur sur des réalités économiques, il ne cherche pas, dans la théorie néoclassique, la vérité sur le fonctionnement du capitalisme libéral et sur ses structures objectives mais la considère, dans ses aspects tant explicitement analytiques qu'implicitement normatifs, comme livrant la quintessence de la philosophie tout à la fois pratique et justificatrice, spontanée et savante, que ce système s'est donné ou dont il a hérité, qui l'anime et dont il veut promouvoir la diffusion. Pour s'exprimer de manière moins substantialiste, l'on peut avancer que le paradigme néoclassique est envisagé par l'auteur comme une description synthétique, venue " après coup » et d'utilisation commode, des principes, croyances, valeurs et motivations, eux-mêmes à l'état pratique, qui constituent l'armature symbolique, dotée d'une réelle efficace, des pratiques des agents économiques « immergés » dans le monde social du capitalisme libéral. Mais la théorie néoclassique, synthèse formalisée des croyances fonctionnelles accompagnant ce système, en est venue à posséder elle-même une efficacité sur son fonctionnement, par 
le système de justification qu'elle lui apporte et par le biais des politiques économiques qu'elle inspire plus que jamais ; l'auteur le souligne :

« The introduction of these ideas into economics gave rise to a number of concepts which have served, consciously or unconsciously, as the foundation of the discipline and shaped the strategy of capitalism. » (Chapra, 1992, p. 27-28)

21 En bref, dans un contexte discursif tout autre, puis qu'essentiellement normatif, l'auteur adopte une démarche intellectuelle qui n'est pas sans rappeler celle de certains anthropologues : sans pour autant adhérer à la conception selon laquelle les «théories indigènes ", parfois déjà " savantes » (au sens où ne les formulent que des spécialistes au sein de la tribu) recèlent la " vérité » sur les pratiques sociales du groupe étudié, ces anthropologues pensent que lesdites théories, qui n'ont que peu en commun avec celles que construit l'anthropologie, constituent une voie d'accès privilégiée à la compréhension de la logique de ces pratiques; et que, par ailleurs, sans être dictées par une "théorie indigène ", ces dernières sont en partie renforcées, dans leur contenu et leur régularité, par la connaissance - souvent partielle - de la théorie.

\section{Les formes de la critique}

Sur la base de cette référence privilégiée à la théorie néoclassique, l'auteur articule selon deux points de vue l'argumentation éthique qui sous-tend sa vision critique de l'Occident économique :

a) selon le premier, son argumentation s'arrête à la seule considération normative des présupposés, principes, fins et moyens que le capitalisme libéral «se donne » (le capitalisme est très souvent substantialisé par l'auteur, par commodité rhétorique et non, apparemment, par position philosophique); il s'agit là d'éléments qui apparaissent comme acceptés ou fixés "au départ " (on parlera par commodité de "prémisses ») et dont la dénomination est reprise des "hypothèses fortes » de la théorie néoclassique, que l'expression d'« individualisme méthodologique » synthétise : l'atomicité des agents économiques, la libre détermination de leurs préférences, le comportement rationnel assimilé à la maximisation de leurs utilités, la non-saturation de leurs besoins, etc. ;

b) selon le second, l'auteur prend en compte, pour les évaluer selon les valeurs de l'éthique qui est pour lui celle de l'islam, les «états du monde social » censés résulter de l'admission des présupposés, de l'application des principes, de la poursuite des fins et de l'utilisation des moyens qui sont ceux du capitalisme libéral.

En fait, s'agissant du premier point de vue d'argumentation évaluative, qui prend appui sur des principes, si nombre des prémisses des actions économiques formant la trame de l'existence du capitalisme libéral entrent en contradiction, en elles-mêmes, avec les valeurs constituant le corpus éthique de l'islam, si l'Occident économique et ses avatars, couvrant l'ensemble de la planète, constituent une "faillite " éthique, c'est d'abord et fondamentalement que ce que l'auteur appelle la worldview of capitalism est le produit d'une longue évolution historique occidentale qui a soustrait le monde socioéconomique au jugement éthique, en produisant une value-free society. Cette vision du monde se donne à lire tout autant dans les grands courants philosophiques apparus au Siècle des Lumières et dans les théories scientifiques nées au XIX ${ }^{e}$ siècle - dont la théorie néoclassique formalisant le libéralisme économique - que dans les idéologies diffuses contemporaines (consumérisme, hédonisme, etc.) qui sous-tendent et justifient 
les comportements quotidiens des agents économiques, comportements spontanément " amoraux », c'est-à-dire implicitement conçus comme non susceptibles de relever du registre des jugements éthiques. La généalogie de cette soustraction fait l'objet d'un long développement (Chapra, 1992, p. 20-23) que l'on examinera dans un premier temps.

Dans l'argumentation envisagée du second point de vue, que l'on peut qualifier de "conséquentialiste», l'auteur évalue les états du monde social produits par le (dys)fonctionnement du capitalisme libéral. La théorie néoclassique affirmant, dans l'une de ses versions au moins, que l'économie de marché réalise simultanément le Bien individuel, le Bien social, l'efficience et l'équité, il examine, d'une part, la définition que cette théorie donne de ces concepts pour montrer que leur acception est, dans ce cadre, tout à fait réductrice par rapport à la définition que l'islam en propose et, d'autre part, il avance que ni le Bien individuel, ni le Bien social, ni l'efficience, ni l'équité, même réduits à leur squelette libéral, ne sont atteints, dans les faits, en économie capitaliste concurrentielle. Le monde "occidental» aura abandonné la recherche du règne des fins éthiques pour la conquête du nouveau monde des harmonies économiques, mais, d'après l'auteur, l'optimum cher à Pareto n'était qu'un miroir aux alouettes.

\section{L'Occident économique : histoire et destinée}

\section{Généalogie de l'oubli économique de la morale ${ }^{11}$}

Le XVIII ${ }^{e}$ siècle constitue, du point de vue de l'auteur - qui reprend bien entendu ici de très nombreuses analyses abondamment citées en note - le siècle de la naissance du sécularisme, c'est-à-dire de l'abandon d'une référence collective et unanime à des réalités transcendantes dans lesquelles la vie sociale doit trouver les fondements ultimes aptes à l'orienter, si ce n'est à l'organiser. La Raison délibérant « librement ", c'est-à-dire, en fait, livrée à elle-même - sans que les philosophes ne puissent réellement s'entendre sur ce qu'est cette Raison - tend à miner la place du religieux dans la gestion des affaires humaines et cette Raison délibère, entre autres, en matière d'éthique (et d'éthique économique en particulier); au moins, dans ce cas, la référence éthique subsiste, comme en témoigne la philosophie kantienne. Mais, concurremment, la vision newtonienne du monde tend à prévaloir comme paradigme d'appréhension du monde social, vite conçu comme un système mécanique. Si le Dieu de Newton, quoique réduit au rôle de "Grand Horloger », a encore la fonction de mettre en mouvement la machine du monde et de constituer la garantie suprême de la pérennité de son ordre, la place de Dieu dans le monde social devient indéterminée. On recherche en effet les principes organisateurs immanents de ce dernier; certains croient les trouver dans le contrat social, qui suppose au moins une action collective et concertée des individus. Mais l'on découvrira bientôt les «lois du marché » conçues, sur le mode mécanique, comme les équivalents sociaux des lois du mouvement, l'intérêt individuel prenant la place de l'attraction universelle; ces « lois économiques » sont objectives, susceptibles d'une explication rationnelle et il est donc subjectivement rationnel, pour les acteurs économiques, de s'y soumettre, de se rendre aux raisons de ces lois, en obéissant à ce que dicte l'intérêt individuel bien compris.

Cette vision du système économique, qui va devenir une des théories de référence du monde social dans son ensemble, est dans sa positivité, déterministe (la proposition de 
base est constative : les hommes "obéissent " très majoritairement à leurs intérêts individuels et l'ordre économique trouve là son fondement) et les théories économiques, sociologiques et psychologiques qui suivront ne feront qu'approfondir ce déterminisme, le marxisme comme le behaviorisme et la psychanalyse. Mais du constat de l'existence de comportements réguliers « objectifs ", l'on passe rapidement à l'appel à se rendre à la raison des lois économiques rationnelles (on parle aussi de « mécanique rationnelle »). La théorie économique va donc prendre un accent normatif et devenir performative. Elle contient en effet en elle-même l'argument le plus apte à convaincre tout un chacun du bien-fondé de l'application, dans la conduite de sa vie sociale, des lois découvertes par la science de l'économie. Dans le cadre du paradigme de la « main invisible ", en effet, la poursuite rationnelle des intérêts individuels privés se traduit à la fois par la maximisation des satisfactions individuelles et par l'optimisation de l'utilité sociale totale, chère aux premiers utilitaristes (Jeremy Bentham et John Stuart Mill), ce qui d'ailleurs confine au truisme, puisque cette utilité totale n'est, dans la première version de l'utilitarisme - reprise par l'École économique de Cambridge (Marshall et Pigou) -que la somme des utilités individuelles. Cette convergence des intérêts individuels et $\mathrm{du}$ "bien public» (réduit à l'utilité sociale ainsi définie) constitue donc un argument définitif de "soumission" individuelle aux lois « objectives » de l'économie.

L'utilitarisme des fondateurs de la doctrine du même nom, dans sa version hédoniste en particulier - et même si elle est présentée de façon plus nuancée dans leurs textes ne pouvait que dégénérer en un matérialisme vulgaire, pour lequel le plaisir matériel, rebaptisé utilité, est le «bien » et la peine est le «mal »: «Utilitarianism reinforced by materialism, provided the logical rationale for single-minded pursuit of wealth and bodily pleasures. » (Chapra, 1992, p. 27)

La maximisation de l'utilité individuelle va donc tenir lieu de seule valeur éthique et la greatest happiness of the greatest number, de seul critère d'appréciation de ce qu'est le meilleur état possible du monde social. Mais ces pseudo-concepts éthiques ne peuvent être d'aucune utilité en vue de la gestion de la vie collective, car il n'est pas possible, rappelle l'auteur, dans un contexte où chacun recherche la maximisation d'utilités dont il est le seul à pouvoir décrire le contenu (des goûts et des couleurs, on ne discute pas) et où aucun consensus n'est envisageable sur ce sujet, de mesurer et d'agréger le bonheur de millions de personne dans le but de comparer avec cette utilité sociale celle que pourrait procurer un état alternatif du monde social. Ce dernier est donc livré à un pluralisme éthique désenchanté et impuissant à en changer collectivement les aspects insatisfaisants que certaines consciences continuent, malgré tout, à percevoir et contre lesquels des révoltes individuelles ou collectives se sont, sans succès, dressées.

31 Toute préoccupation éthique individuelle, y compris fondée dans une croyance religieuse, n'a en effet pas totalement disparu, écrit l'auteur, loin de là. Mais, dans une société sécularisée, l'adhésion à une croyance religieuse et aux valeurs éthiques qu'elle fonde est elle-même devenue affaire de préférences individuelles et privées, selon le langage des économistes, préférences qui sont des variables " exogènes » du système, toujours selon le même langage. Libre à chacun, d'ailleurs, de maximiser ses utilités personnelles en accomplissant des actions qui soient, d'après sa croyance personnelle ou d'après une croyance religieuse collective, éthiquement bonnes, si cet accomplissement lui procure ainsi le bonheur optimal (c'est-à-dire, possède le meilleur coût d'opportunité : il n'est pas d'action coûtant le même effort qui puisse lui apporter 
plus de bonheur)! C'est ce caractère facultatif, laissé au libre choix de chacun, de la poursuite de fins éthiques dont la détermination relève elle-même du libre choix, qui apparaît caractéristique d'un monde économique capitaliste disembedded (désenclavé, autonomisé), pour reprendre le terme de K. Polanyi, cité par l'auteur qui écrit par ailleurs : "Moral values lost their collective sanction and collective value judgements became an anathema. »(Chapra, 1992, p. 24)

La soustraction à toute possibilité de jugement éthique collectif de la sphère économique (se soumettant tendanciellement les autres sphères sociales), processus pleinement abouti avec la rediffusion d'un capitalisme libéral actuellement en plein revival, constitue donc pour l'auteur - qui rejoint ici de nombreux économistes et philosophes occidentaux, abondamment cités par lui et appartenant à diverses courants dont certains fort éloignés de ceux habituellement désignés comme conservateurs -ce produit de l'enchaînement ou de la convergence, selon les cas, de multiples évolutions internes à la sphère "idéologique » et s'y déroulant selon une logique propre, plus que sous l'influence des transformations du système économique lui-même (l'on ne s'étonnera pas que l'auteur ne pratique pas une histoire matérialiste des visions du mondes). La worldview du capitalisme libéral est ici conçue plus comme une constellation d'idéologies en correspondance fonctionnelle avec ce dernier qu'une émanation directe de celui-ci; elle en facilite cependant par ce fait-même la "reproduction" et l'auteur insiste sur la diffusion de ces idéologies, devenues philosophies spontanées majoritaires, en Occident et ailleurs, et cite à ce propos un extrait de Small is Beautiful! de E. F. Schumacher : « ... these nineteenth century ideas are firmly lodged in the minds of practically everybody in the western world today, wether educated or uneducated. "(Chapra, 1992, p. 24)

Rien n'incarne mieux pour l'auteur l'amoralisme de cette nouvelle conception du monde social que le second paradigme emprunté par certains théoriciens sociaux sur " sciences dures " (après la mécanique newtonienne) pour penser-et justifier le monde du capitalisme concurrentiel: celui de la doctrine darwinienne où la société de concurrence est pensée sur le mode de la lutte pour la survie, avec sélection des «plus aptes».

La value free society, selon l'expression de l'auteur, n'est donc pas une société totalement amorale, mais une société dont les différentes sphères, et en premier lieu la sphère économique, ont été progressivement soustraites aux jugements relevant d'une éthique réellement collective, dont l'existence requiert un consensus sur des valeurs essentielles et un fondement dans la référence à la transcendance. Les branches de la généalogie de cette soustraction, globalement dénommée sécularisation, ont pour nom physicalisme (l'importation du paradigme newtonien), déterminisme, utilitarisme, individualisme, matérialisme, pluralisme (mais aussi pragmatisme, positivisme et darwinisme social) et finalement athéisme. L'auteur résume ainsi son propos:

"Since the socio-economic importance of religion essentially lies in the collective sanction it provides to moral values, thereby ensuring their unchallenged acceptance as a basis for socio-economic and political decisions, the loss of the religious sanction for values was a great tragedy. Society became deprived of the socially-agreed filter mechanism... Even though the individual conscience... may still be there to serve as a filter mechanism at the individual level, it is not sufficient to perform the function ofa socially-agreed filter mechanism which is needed to create a harmony between individual self-interest and social interest... Given the reluctance to use the filter mechanism provided by morally sanctioned value judgments, and the weakening of the feeling of social obligation that religion 
intensifies, it is not possible to realize the dream of a society... where scares resources are used not only to fulfill the needs of all but also to create an equitable distribution of income and wealth... moral uplift and social solidarity are not possible without the moral sanction that religion provide. "(Chapra, 1992, p. 24-25)

Avec la sécularisation, ce n'est pas tant «le mécanisme de filtrage éthique collectivement accepté » lui-même qui disparait, dont l'auteur -qui ne raisonne à aucun moment en terme d'âge d'or religieux des origines ${ }^{12}$ et de décadence séculière -ne prétend nullement qu'il ait été historiquement utilisé et ait permis l'existence d'une société d'avant le « désenchantement du monde » économiquement plus conforme aux valeurs de justice et d'équité ; ce qui disparaît, c'est surtout l'exigence religieusement fondée du recours à ce "mécanisme » indispensable, que la religion rendait également disponible sous la forme de valeurs éthiques essentielles qu'elle présentait comme universelles. L'auteur mentionne, parmi les tentatives « artificialistes » de construction séculière de l'équivalent d'un tel mécanisme de filtrage, visant à l'universalité, celle que J. Rawls expose dans A Theory of Justice, et connue sous l'expression de principe du "voile d'ignorance »; mais, sans que l'auteur ne se prononce sur les « résultats » de cette construction (il est difficile de penser qu'il soit insensible au premier volet, dit " principe de différence », du second principe ${ }^{13} \mathrm{il}$ écrit : « me veil of ignorance... is no more than wishful thinking and cannot be of any practical use in deriving " just " principles for social organisation. » (Chapra, 1992, p. 26)

Démuni de ce filtre et de l'exigence-même d'en appliquer un, l'Occident est donc livré ( $d$ 'après les théoriciens néoclassiques qui ont produit une impressionnante synthèse, appliquée à leur objet, des idéologies et doctrines aux noms en « isme » énumérées plus haut) à l'univers des harmonies économiques que garantit le marché autorégulateur, dont les présupposés, les principes, les fins et les moyens qu'il impose aux agents et qui lui sont inhérents, relèvent, de droit, d'un ordre coupé de toute préoccupation éthique et qui a vocation à s'étendre à l'ensemble des sphères de la vie sociale. Si ces présupposés, principes, fins et moyens sont perçus, par quiconque se soucie encore d'éthique sociale, comme globalement inconciliables non seulement avec les valeurs prônées par les grandes religions mais encore avec celles, sécularisées, que proposent les systèmes de philosophie morale rationaliste, ce n'est donc pas parce qu'ils ont été choisis et évalués à l'aide d'un « filtre éthique » différent de ceux qui ont été construits par ces grandes religions et ces philosophies, mais parce qu'ils n'ont été ni réellement choisis ni éthiquement évalués.

\section{La worldview prise au mot}

L'auteur n'en reste cependant pas à cette vision généalogique critique de l'Occident économique, il tente aussi d'évaluer son état contemporain. Ce qu'il pourrait faire en le passant à son propre « filtre éthique ». Mais la «stratégie discursive» de l'auteur est autre. Il constate d'abord que la worldview du capitalisme libéral, telle que la livrent les théoriciens néoclassiques qui en sont à la fois les interprètes et les porte-parole - voir les apologistes - affirme que l'économie de marché réalise simultanément et sous certaines conditions, le Bien individuel, le Bien social, l'efficience et l'équité. Sa stratégie consistera à "prendre » au mot la worldview de l'Occident économique et à montrer que les quatre catégories énoncées ne peuvent être définies, dans le cadre de la théorie néoclassique, que de manière tout à fait réductrice, en particulier par rapport au contenu éthique que leur donne les textes canoniques de l'islam et les 
interprétations, y compris modernistes, de ces derniers. La réalisation du Bien individuel, du Bien social et de l'équité, ainsi réduits à leur «squelette libéral », quand bien même elle serait effective - ce qu'elle n'est pas, comme le montre amplement l'auteur en reprenant les arguments, désormais classiques, développés par les critiques du capitalisme libéral (Chapra, 1992, p. 34-53) - ne répondrait guère aux critères éthiques développés, tant par l'islam que par les philosophies éthiques universalistes autres que les versions les plus « brutales » de l'utilitarisme.

L'auteur part de cette affirmation de la réalisation simultanée du Bien individuel et du Bien social dans le cadre d'hypothétiques états d'équilibre général, atteints, moyennant le « respect » d'un corps d'hypothèses «lourdes », par le biais du mécanisme des prix, qui constitue ce qu'il appelle un value neutral filter mechanism. Ce mécanisme assurerait l'allocation la plus efficiente des ressources (critère économique) et la distribution la plus équitable des richesses (critère éthique), sans qu'il soit besoin d'interventions rectificatrices ou compensatrices ex post ; ces dernières sont mêmes à éviter, surtout si elles ne visent pas des objectifs strictement économiques et découlent de jugements de valeurs extérieurs à la théorie économique; elles ne peuvent en effet alors que déclencher des effets pervers qui renforceront les déséquilibres du cycle suivant (seules sont admises les interventions qui visent à assurer le "respect» de conditions strictement économiques de départ, jugées saines par la théorie néoclassique).

Pour l'auteur, qui se situe dans une position que l'on pourrait dire « réformiste », les mécanismes réels du marché réel génèrent des états du monde socio-économique qui devraient pouvoir faire l'objet, après une analyse relevant d'une théorie économique positive, de jugements de valeur (opposés à jugements de vérité) formulés à partir de points de vue globalement consensuels, par définition extérieurs à la théorie économique positive, le jugement sur l'efficience ne pouvant être formulé que sur la base d'une théorie normative de la rationalité économique qui se donne comme telle, et le jugement sur l'équité à partir d'une théorie éthique de nature clairement philosophique (qu'elle soit rationaliste ou qu'elle ait recours à des fondements d'ordre transcendant). Pour les théoriciens néoclassiques, affirme l'auteur, ce sont les résultats théoriques du modèle décrivant le parfait fonctionnement de l'économie de marché qui servent d'étalon d'efficience et d'équité permettant de juger de l'état du monde socioéconomique réel. Or ces résultats théoriques consistent en états d'allocation des biens et de distribution des richesses qui découlent du fonctionnement théorique du modèle, le premier type d'états étant censé réaliser et, par ce fait-même, définir l'efficience, et le second, réaliser et définir l'équité. La théorie néoclassique de l'équilibre général contiendrait donc en elle-même deux théories normatives qu'elle n'énoncerait pas comme telles : l'une, concernant la rationalité économique permettant de juger de ce qu'est en soi l'efficience et l'autre, de type éthique, définissant ce que sont l'équité, le Bien individuel et le Bien collectif. Ces deux théories devraient donc être extraites de la théorie économique positive, autonomisées par rapport à elle et, de plus, clairement découplées. D'une part, des états d'efficience économique, au sens d'allocation la plus rationnelle des ressources (selon une définition normative de la rationalité), pourraient être définis ; d'autre part, des définitions éthiques de l'équité et des Biens, individuel et social pourraient être discutées.

40 Certes, comme le montre très bien J.-P. Dupuis (1992, p. 47-73), dont les propos peuvent éclairer ceux de l'auteur, la distinction de l'efficience et de l'équité est un "progrès ", qui n'a pu intervenir qu'après une rupture avec le premier utilitarisme pour lequel seul 
comptait la somme totale des utilités individuelles, dite utilité sociale et qui ne se souciait donc nullement de problèmes de distribution, encore moins d'équité. C'est Walras et surtout Pareto qui sont les premiers à avoir formulé à la fois les problèmes d'efficacité dans l'allocation des ressources et de justice dans la distribution des richesses. Mais, d'après l'auteur, l'optimum de Pareto, en pratiquant le couplage efficience-équité, permit la construction d'une (pseudo)éthique économique endogène qui posa une définition ad hoc de l'équité constituant en fait la négation de toute démarche éthique :

"Since the resultant configuration) ${ }^{14}$ of goods and services is a reflection of consumer preferences, it is the most "efficient" - it is not possible to improve upon it. Since this configuration also determines the income earned bythe respective factors of production on the basis of their contribution to output and revenue, the resultant income distribution is also «equitable... The market system... determines... also the most «equitable» distribution of income in a rational and impartial manner without value judgments. »(Chapra, 1992, p. 19)

41 Le postulat, considéré comme fondement de cette éthique économique endogène, selon lequel la distribution des revenus au prorata de la contribution des facteurs à la production des output est en elle-même équitable, n'est en effet nullement mis en cause, alors que c'est précisément lui qui doit constituer l'objet de discussions par toute théorie d'éthique économique autonome, de même que le dogme issu de la proposition centrale de l'optimum selon laquelle il ne peut être question de réduire, même légèrement, le bien-être des mieux pourvus pour améliorer celui des moins bien lotis.

C'est l'idée même d'une éthique qui serait ainsi «scientifiquement fondée » que refuse l'auteur, pour qui la proposition selon laquelle la main invisible du marché pourrait garantir le comportement éthique des agents économiques constitue la négation-même de l'éthique. Mais inversement, aucune "réforme éthique " ne sera accomplie tant qu'elle n'aura pas pénétré les structures économiques pour y instaurer la justice économique ou équité (à définir, ce que l'auteur ne fait pas véritablement) dans la répartition des revenus et richesses, notions clés dont aucune doctrine économique ne peut dicter de définition mais dont il est capital, d'après l'auteur, qu'il en existe une, reconnue de tous et donc, dans ses vues, fondée religieusement, en l'occurrence sur le principe islamique de la 'adâla, à (ré)interpréter.

Le fait que, parti de cette notion et de quelques autres principes peu nombreux, conçus par lui comme fondamentaux et retraduits en langage économique contemporain, l'auteur, en tant $\mathrm{qu}^{\prime}$ «économiste islamique» prenant au sérieux ce que cette expression signifie pour lui, ait pu effectuer ce périple intellectuel, critique non pas tant de l'Occident géographique en tant que tel, que de l'Occident devenu " planétaire " dans ses traits économiques essentiels et communs, contribuera peut-être à montrer que la production de discours d'Économie islamique de type universitaire ne constitue en rien, sociologiquement, une tentative de "retour» à des temps mythifiés de "pureté économique", ou à une volonté de se couper d'un Occident "maudit»; l'Occident économique est en effet, aux dires-mêmes de l'auteur, maintenant partout, et l'on ne peut que se confronter à ses théories, en reprenant, de manière critique le langage économique qu'il a créé, pour en transformer les réalités économiques. 


\section{BIBLIOGRAPHIE}

ARROW K.J., (1951) 1963, Social Choice and Individual Values, New York, Wiley.

BLAUG M., 1982, La méthodologie économique, Paris, Economica.

BROCHER H., 1995, «L'économie comme science positive et normative », dans d'AUTUME A. et

CARTELIER J., L'Économie devient-elle une science dure ?, Paris, Economica.

CHAPRA M. U :

-1985, Towards a Just Monetary System, London, The islamic Foundation.

-1992, Islam andthe Economic Challenge, Kano (Nigeria), Herndon (VA, USA), The islamic Foundation andThe International Institute of islamic Thought.

CHOUDHURY M. A., 1992, The Principles of islamic Political Economy, a Methodological Enquiry, New York, London, St. Martin Press, Macmillan.

CHOUDHURY M. A. et MALIK U. A., 1992, The Foundations of islamic Political Economy, London, Macmillan.

DUPUIS J.-P., 1992, Le sacrifice et l'envie. Le libéralisme aux prises avec la justice sociale, Paris, Calmann-Lévy.

SENA. K., 1970, Collective Choice and Social Welfare, San Francisco, Holden-Day.

\section{NOTES}

1. Cité dans Brochier, 1995, p. 38 ; voir aussi Blaug, 1982, p. 109-147. Il est à noter que l'Économie est la seule des sciences sociales à développer et assumer ce volet normatif, du moins dans certains de ses courants théoriques, en particulier ceux dont les membres visent la constitution de ce qu'ils dénomment une «Économie pure» (terme vieilli) ou «la» Science Économique, tendant au statut de "science dure » et nient, au moins implicitement, l'appartenance de cette dernière à l'ensemble des sciences sociales (l'Économie normative se voyant alors attribuer lé statut de «science appliquée »).

2. Beaucoup d'« économistes islamiques » font remarquer, d'un point de vue normatif, qu'il ne faut pas confondre les structures économiques islamiques (dont certains contestent qu'il en existe véritablement dans le faits) et les structures économiques des pays islamiques (i. e., dont la population est en majorité musulmane et, le cas échéant, dont l'islam est religion d'État).

3. Cf., par exemple, les références citées en note 1 .

4. Il ne peut être question d'aborder ici cet aspect, qui mériterait à lui seule une recherche spécifique.

5. Le doctorat (Ph. D) de celui qui est l'un des plus «productifs» d'entre eux, M. A. Choudhury, s'intitule : Some aspects of Optimal Human Capital lnvestmentand Economic Growth: a Theoretical and Empirical Analysis.

6. Il s'agit d'une part de Masudul Alam Choudhoury (1992) et du même auteur, avec Uzir Abdul Malik (1992) et de M. Umer Chapra, d'autre part, (1985 et 1992) ; ce dernier ouvrage, intitulé islam and the Economic Challenge constitue l'objet essentiel de l'analyse de discours menée infra.

7. Outre dans des revues spécialisées en économie et sciences sociales islamiques (Review of islamic Economics, Journal of Research in islamic Economies, Journal of islamic Banking and Finance (Karachi), Thoughts on Economies (islamic Economies Research Bureau, Dacca), Journal of islamic 
Economies (Kuala Lumpur), American Journal of islamic Social Sciences, (Journal of Economie Cooperation Among islamic Countries), ces économistes publient des articles sur divers thèmes d'Économie islamique dans de grandes revues économiques non strictement «orthodoxes " sur la plan théorique (International Journal of Social Economies, Journal of Development Studies, International Journal of Manpower, Journal of Social Sciences, Journal oi Economie Development, etc.) ou dans des revues scientifiques de diffusion nationale sans préoccupations "islamiques » particulières (Criterion (Karachi), Theory and Practice (Karachi), Journal of Rural Development and Administration (Peshawar), The Kashmir Economie Review (Muzaffarabad, Pakistan).

8. La notion d'homo economicus et l'individualisme méthodologique «élargi " (supposant l'atomicité des "acteurs", la rationalité maximisatrice (rational choice) et l'utilitarisme) sont critiqués comme concepts et cadre analytiques de base duparadigme néoclassique (les auteurs se rattachant plutôt sur le plan théorique aux courants institutionnalistes). Ils sont aussi, bien évidemment, invalidés comme idéaux normatifs (ce qu'ils sont dans le paradigme néoclassique qui n'est jamais purement positif), inconciliables avec les principes éthico-économiques de l'islam tel qu'il est interprété par ces auteurs.

9. L'on ne peut développer ici les réalisations concrètes assurant, d'après l'auteur, l'existence de ces conditions; ce dernier ne fait qu'en esquisser les grandes lignes. Elles supposent un consensus, obtenu par la délibération, sur les valeurs sociales essentielles à promouvoir et sur le système des préférences individuelles à satisfaire prioritairement par l'appareil économique; ce qui implique l'existence de ce que l'auteur dénomme un socially-agreed ethical filter mechanism, qui n'invalide pas la poursuite des intérêts individuels ni le rôle du marché et du système des prix dans l'allocation des ressources et des biens, mais qui limite leur marge de manoeuvre, filtre dont l'idéal est qu'il soit volontairement accepté et intériorisé par chacun sur la base des valeurs ethico-religieuses qui le fondent, mais dont un État à la fois fort, démocratiquement élu et démocratiquement contrôlé, peut venir renforcer l'efficacité, au cas où des groupes visant la satisfaction prioritaire de leurs propres intérêts viendraient à le contourner. Une citation semble éclairante sur ce point (Chapra, 1992, p. 213) : «It is evident that, unlike both capitalism and socialism, the goals of islam are absolute and a logical outcome of its underlying philosophy. They do not constitute a hotchpotch of incongruous elements resulting from the struggle for survival and domination between pluralist groups or social classes». Dans ses travaux, l'économiste M. A. Choudhury propose une islamic social welfare function with ethical endogeneity, qui peut être maximisée puisqu'elle suppose un accord collectif sur les préférences essentielles à satisfaire et contourne ainsi le problème insoluble de l'agrégation des préférences individuelles et de leur comparaison interpersonnelle, obstacle sur lequel la New Welfare Economics, avec la social welfare function de Bergson, (reprise dix ans plus tard par Samuelson dans ses Foundations of Economic Analysis) a buté et qu'a mis en lumière la Théorie des choix sociaux et le théorème d'impossibilité de Arrow (1963). Choudhury rejoint ainsi, sur d'autres bases, A. Sen (1970) dans son affirmation de la possibilité, sous certaines conditions strictes, de construire une fonction d'utilité collective. On peut constater, au passage, la pleine inscription dans les débats économiques contemporains de la version, examinée ici, de l'Économie islamique.

10. La contester radicalement pourrait consister, par exemple, comme l'ont fait des économistes d'inspiration marxiste, à montrer explicitement qu'elle constitue un cadre d'analyse inadéquat à son objet, construit à l'aide de notions idéologiques en réalité normatives, empruntées aux représentions pratiques et justificatrices des agents économiquement dominants. L'auteur n'affirme pas non plus, comme le font les fermes opposants au paradigme néoclassique, que ce dernier, loin de permettre l'analyse « positive » de ce qui n'est qu'un système économique parmi d'autres, en constitue en réalité une apologétique déguisée, dans la mesure où il ne le désigne pas comme système historiquement situé et comportant ses caractéristiques propres, mais comme incarnation d'une rationalité économique supposée universelle et anhistorique. 
11. Cet intitulé s'inspire librement de celui d'un article de J.-N. Ferrié («Justice et ajustement: l'oubli économiciste de la morale libérale», dans Âge libéral et néolibéralisme, Vie Rencontres franco-égyptiennes de Science Politique, CEDEJ, Le Caire, 1996).

12. Il est à noter que, contrairement à de nombreux ouvrages concernant un système économique islamique à construire, islamic and the Economie Challenge ne comporte aucune référence à une société islamique des origines (i.e., du temps du Prophète Muhammad ou des premiers califes «bien guidés») qui aurait intégralement incarné, sur le plan socio-économique, les valeurs essentielles de l'islam, et dont la reconstitution est, au yeux des meilleurs historiens musulmans eux-mêmes, des plus périlleuses. Il n'est question dans cet ouvrage de Chapra que des enseignements du Prophète et non de l'état de la société de son temps.

13. Principe selon lequel, d'après la formulation de J.-P. Dupuis (1992, p. 139), « les inégalités sociales et économiques doivent être organisées de façon à ce que... elles apportent aux plus désavantagés les meilleures perspectives...».

14. Il s'agit de la configuration résultant du mécanisme des prix.

INDEX

Mots-clés : économie, économie islamique, éthique, Occident

\section{AUTEUR}

FRANÇOIS IRETON

Cedej 